\title{
Using the Contralateral Reverse Less Invasive Plating System for Subtrochanteric Femur Fractures in Elderly Patients
}

\author{
Yuanming Ouyang Yongping Wang Cunyi Fan Zhongtang Liu \\ Sheng Liu Fenfen Li \\ Department of Orthopedic Surgery, Shanghai Sixth People's Hospital, Shanghai Jiaotong University, \\ Shanghai, China
}

\section{Key Words}

Subtrochanteric fracture $\cdot$ Less invasive stabilization system $\cdot$ Minimally invasive treatment $\cdot$ Osteoporosis

\begin{abstract}
Objective: In this retrospective study, we evaluated the efficacy of the reverse less invasive plating system (LISS) in the management of subtrochanteric fractures (STF) in elderly patients that are expected to be difficult to nail. Subjects and Methods: Fifty-five patients with STF were treated from April 2005 to December 2007. Of these, 26 (16 females and 10 males, age $>65$ years, average $79.8 \pm 3$ ) had nonpathologic fractures that were internally fixed with a LISS device. The average follow-up period was 24 months (range 15-30). The main outcome measures evaluated were pain, union, varus deformity and implant failure. Radiographic and clinical evidence of functional outcome and complications were also evaluated. Results: Mean perioperative blood loss was $120.5 \pm 12 \mathrm{ml}$ (range 65-285), and mean postoperative hospital stay was $7.8 \pm 2$ days (range $4-14$ ). Using the reverse LISS plates led to complete union of STF in all of the $26 \mathrm{el}-$ derly patients without additional procedures. Conclusion: Due to advantages like high union rate, early postoperative mobilization and short operation time, the reverse LISS device offers an alternative management of STF in elderly patients unsuitable for nailing procedures.
\end{abstract}

Copyright $\odot 2012$ S. Karger AG, Basel

\section{KARGER}

Fax +4161306 1234

E-Mail karger@karger.ch

www.karger.com (c) 2012 S. Karger AG, Basel

1011-7571/12/0214-0334\$38.00/0

Accessible online at:

www.karger.com/mpp

\section{Introduction}

Subtrochanteric fractures (STF) are the least common fractures of the proximal femur but pose considerable challenge to orthopedic surgeons. Given the ageing population and a rising incidence of hip fractures, a rise in the number of STF in the older age group is expected to occur. In elderly patients, STF occur with low-energy trauma, as in a simple fall. Frequently, these patients have osteoporotic bones with thin cortices, particularly women, but there is little soft-tissue damage or extensive comminution, and the muscular forces are generally weaker [1]. However, there may be preexisting medical ailments and ambulatory problems.

Intramedullary nails have been considered the technique of choice for treating simple subtrochanteric femoral fractures throughout the literature [1]. However, various problems are associated with the application of intramedullary nails. Various complications have been encountered in comminuted fractures, such as nonunion, delayed union, varus deformity, peroneal nerve paralysis, shaft fracture during surgery, fracture of the trochanter major, perforation in the femoral neck or knee joint and

Y. Ouyang and Y. Wang contributed equally to this paper.

Cunyi Fan

Department of Orthopedic Surgery, Shanghai Sixth People's Hospital

Shanghai Jiaotong University

600 YiShan Road, Shanghai 200233 (China)

Tel. +861380183 2808, E-Mail fancunyi888@hotmail.com 
Table 1. Preoperative data of the 26 STF patients

\begin{tabular}{lc}
\hline Variables & Number \\
\hline Male/female & $10 / 16$ \\
Mean age, years & 79.8 \\
Seinsheimer classification & \\
Type I & 0 \\
Type II & 12 \\
Type III & 7 \\
Type IV & 3 \\
Type V & 4 \\
Mechanism of injury & \\
Traffic crash & 4 \\
Fell down stairs & 6 \\
Fell while walking & 12 \\
Direct blunt trauma & 4 \\
With associated injury, patients & 12 \\
Proximal humerus fracture & 4 \\
Bimalleolar ankle fracture & 2 \\
Fracture of the acetabulum & 1 \\
Fracture of the forearm & 6 \\
Fracture of the calcaneus bone & 2 \\
Comorbidity, patients & 23 \\
Neurological disease & 2 \\
Hypertension & 8 \\
Cardiac insufficiency & 4 \\
Diabetes mellitus & 5 \\
Pulmonary disease & 3 \\
Thyroid carcinoma & 1 \\
\hline
\end{tabular}

fixation device breakage [2]. Furthermore, nailing is often unsuitable for some special situations, such as fracture patterns with comminution and presence of a posteromedial butterfly fragment, STF with extension into the piriform fossa, femurs with narrow medullary canals, and severely deformed femurs or osteoporotic bones with thin cortices. In these situations, extramedullary internal fixation is recommended. Traditional open plating is associated with higher rates of delayed union and nonunion and an elevated infection rate. Because of these factors, alternative means of treating STF have been the subject of debate for a considerable time.

According to the principles of 'minimally invasive' surgery, the less invasive plating system (LISS) has been effectively used in the management of complex distal femoral fractures with satisfactory results. The LISS is an extramedullary internal fixation system which combines the advantages of both interlocked intramedullary nailing techniques and the early advances of the so-called biological plating technique into one system. Thus, its biome- chanics are inherently different from conventional plates. Lewis and Ashcroft [3] reported using reverse LISS plating for proximal segmental femoral fractures of the contralateral extremity in polytrauma patients. Reverse LISS plates were used 'upside down' for stabilizing injured femurs. It has been shown that the reverse LISS technique can reduce operative complications and shorten operating time. To our knowledge, this is the first article to focus on the use of LISS for the fixation of STF in elderly patients.

In the present study, a series of STF in elderly patients treated by reverse LISS technique at our institution were reviewed, to determine the suitability of this technique.

\section{Subjects and Methods}

From April 2005 to December 2007, 55 patients with STF were treated at our institution. All of these patients with STF underwent the reverse LISS procedure. The inclusion criteria were: patients with osteoporosis $(\mathrm{n}=10)$, STF with extension into the piriform fossa $(n=5)$, patients with narrow medullary canals $(\mathrm{n}=7)$, and osteomyelitis sequelae with severely deformed femurs $(n=4)$. To confirm osteoporosis as defined by the World Health Organisation [4], formal bone mineral density [4] was performed in all the patients. The exclusion criteria were: a pathologic fracture, a previous hip fracture, and a soft-tissue infection at the fracture site. The preoperative data are summarized in table 1 . There were 16 females and 10 males (age range 65-98 years). Of the 26 fractures, 22 were stabilized within 3 days after injury, 4 more than 1 week after admission, secondary to the treatments for associated injuries and comorbidity. The most common causes of the injuries were a fall at ground level, which occurred in 12 patients. The other causes included traffic accidents: $\mathrm{n}=4$; direct blunt trauma: $\mathrm{n}=4$, and falling down stairs: $\mathrm{n}=6$. All patients were evaluated preoperatively with standard anteroposterior (AP) radiographs of the pelvis, and AP and lateral views of the affected hip. The Seinsheimer classification was used [5]. Twenty-six patients were classified according to types as type II: $\mathrm{n}=12$; type III: $\mathrm{n}=7$; type IV: $\mathrm{n}=3$, and type $\mathrm{V}: \mathrm{n}=4$. Twelve patients sustained 15 additional fractures at locations other than the STF during the study period. Twentythree patients had one or more concomitant medical conditions unrelated to the injury such as hypertension and cardiac insufficiency. Closed fracture reduction was achieved indirectly using the traction under image intensifier control and in 8 cases also directly with a Schanz screw inserted percutaneously in the proximal main fragment to correct the remaining flexion malalignment. Traction and rotation were used to achieve and maintain reduction during the surgical procedure, which was verified through AP and lateral views under image intensifier.

The patient was placed on a traction table and was operated under either general or spinal anesthesia. A small incision (4$6 \mathrm{~cm}$ ) was made on the level of the greater trochanter after basic fracture reduction (fig. 1). For spiral fractures, percutaneous interfragmentary screw fixation was performed without opening the fracture site. An appropriate-hole LISS plate was inserted along the femoral shaft from proximally to distally beneath the muscle tis- 
sue without stripping the periosteum of the lateral femur. As usual, 9 and 13 holes were used and a contralateral-side LISS plate was used 'upside down' for stabilization of the STF. With the aid of an insertion handle and with an image intensifier, the LISS plate was placed in optimal position. To confirm the position, frontal and rotational alignment and leg length were checked. If there was any malalignment, rotational deformity or limb-length discrepancy, reduction was repeated. Subsequently, the plate was temporarily fixed to the bone with Kirschner wires; 4 mono- or bicortical fixed-angle screws were used in the shaft (distal) area according to the age and bone quality of the patients. Optimal placement and screw length was checked radiographically. Finally, the incisions were irrigated and closed over without drainage. The whole process of stabilization was finished within $45 \mathrm{~min}$. To ensure optimal placement of the plate on the femur, the position of the LISS was checked again radiographically. The self-drilling and self-cutting screws were then inserted through the trocar sleeve and via the stab incisions, using the target device. Generally, 4-6 locking screws were placed into the proximal component of the fracture.

All patients received perioperative prophylactic antibiotics for 1 day. Active exercise of the quadriceps and early ambulation were encouraged. Patients began walking using crutches or a frame with partial weight bearing for approximately 6 weeks. Progressive weight bearing was encouraged. Full weight bearing was encouraged after 3-6 months of partial and increasing weight bearing, based on the degree of comminution of the fractures and evidence of callus formation on radiographs. The patients were reviewed both clinically and radiographically until fracture union. Generally, the patients underwent a standardized followup examination at monthly intervals for the first 6 months, followed by assessment every 3 months. The follow-up examination lasted for a minimum of 15 months.

\section{Results}

All patients attended follow-up examinations for a minimum of 15 months. The average follow-up period was 24 months (range 15-30). The mean age was $79.8 \pm 3$ years (range 65-98), and patients included 16 females and 10 males. No patients were lost to follow-up or died during the period of follow-up. The mean operating time was 45 $\pm 5 \mathrm{~min}$ (range 25-75), and the mean fluoroscopy time was $120 \pm 10 \mathrm{~s}$ (range 85-190). Mean perioperative blood loss was $120.5 \pm 12 \mathrm{ml}$ (range 65-285), and the mean postoperative hospital stay was $7.8 \pm 2$ days (range $4-14$ ). The median incision length through which the plate was inserted was $5.5 \pm 0.5 \mathrm{~cm}$ (range $2.5-8.0$ ). Pain was absent in 15 patients, mild in 7 , and moderate in 4 . In clinical evaluations, the mean Harris hip score was 78.9. Harris hip scores were very good in 6 patients, good in 14 patients, moderate in 5 patients, and poor in 1 patient. The mean collodiaphyseal angle at early postoperative assessment and the last assessment was $136 \pm 2^{\circ}$ (range $\left.131-140^{\circ}\right)$ and $134 \pm 2^{\circ}\left(\right.$ range $\left.129-138^{\circ}\right)$, respectively. The mean collo-
Table 2. Intraoperative and postoperative data of the 26 STF patients

\begin{tabular}{lc}
\hline Variables & Number (range) \\
\hline Anesthetic method & \\
$\quad$ General & 18 \\
$\quad$ Spinal & 8 \\
Operation time, min & $45(25-75)$ \\
Fluoroscopy time, s & $120(85-190)$ \\
Blood transfusion, units & $0.5(0-1)$ \\
Hospital stay, days & $7.8(4-14)$ \\
Median incision, cm & $5.5(2.5-8.0)$ \\
Follow-up time, months & $24(15-32)$ \\
Mean collodiaphyseal angle difference, degrees & $1(0-3)$ \\
Visual analogue scale & \\
$\quad$ Pain absent & 15 \\
Mild pain & 7 \\
Moderate pain & 4 \\
Harris hip score & 78.9 \\
$\quad$ Very good & 6 \\
$\quad$ Good & 14 \\
Moderate & 5 \\
Poor & 1 \\
\hline
\end{tabular}

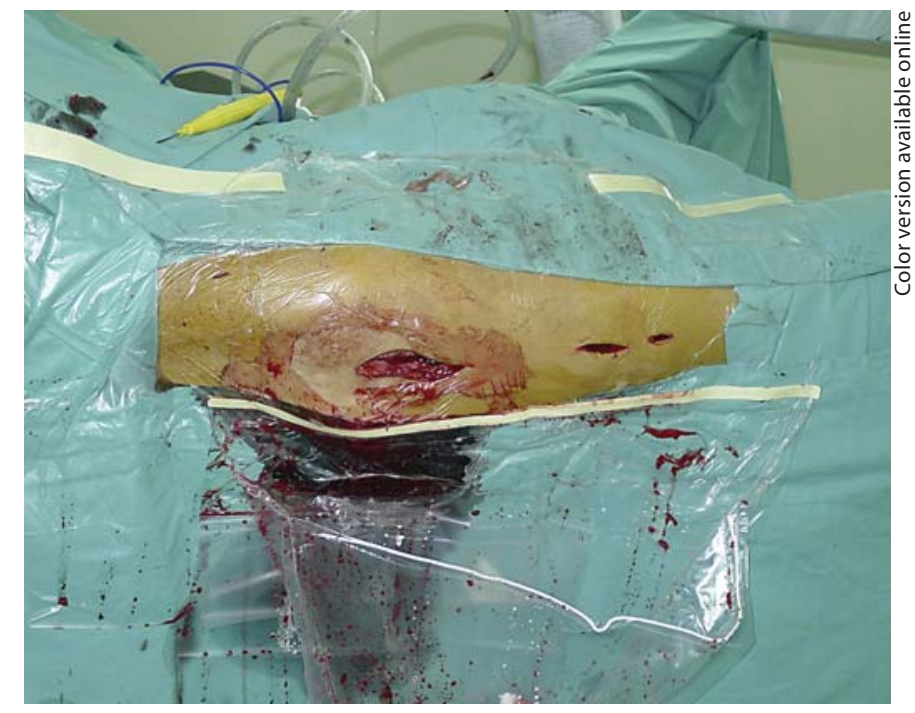

Fig. 1. Separate proximal and distal incisions.

diaphyseal angle difference was 1 (range 0-3) (table 2). All patients achieved fracture union at a median of 4 months (range 3-15 months) without additional procedures. Deep vein thrombosis or pulmonary embolus was not observed. No deaths occurred in this study. No superficial or deep 

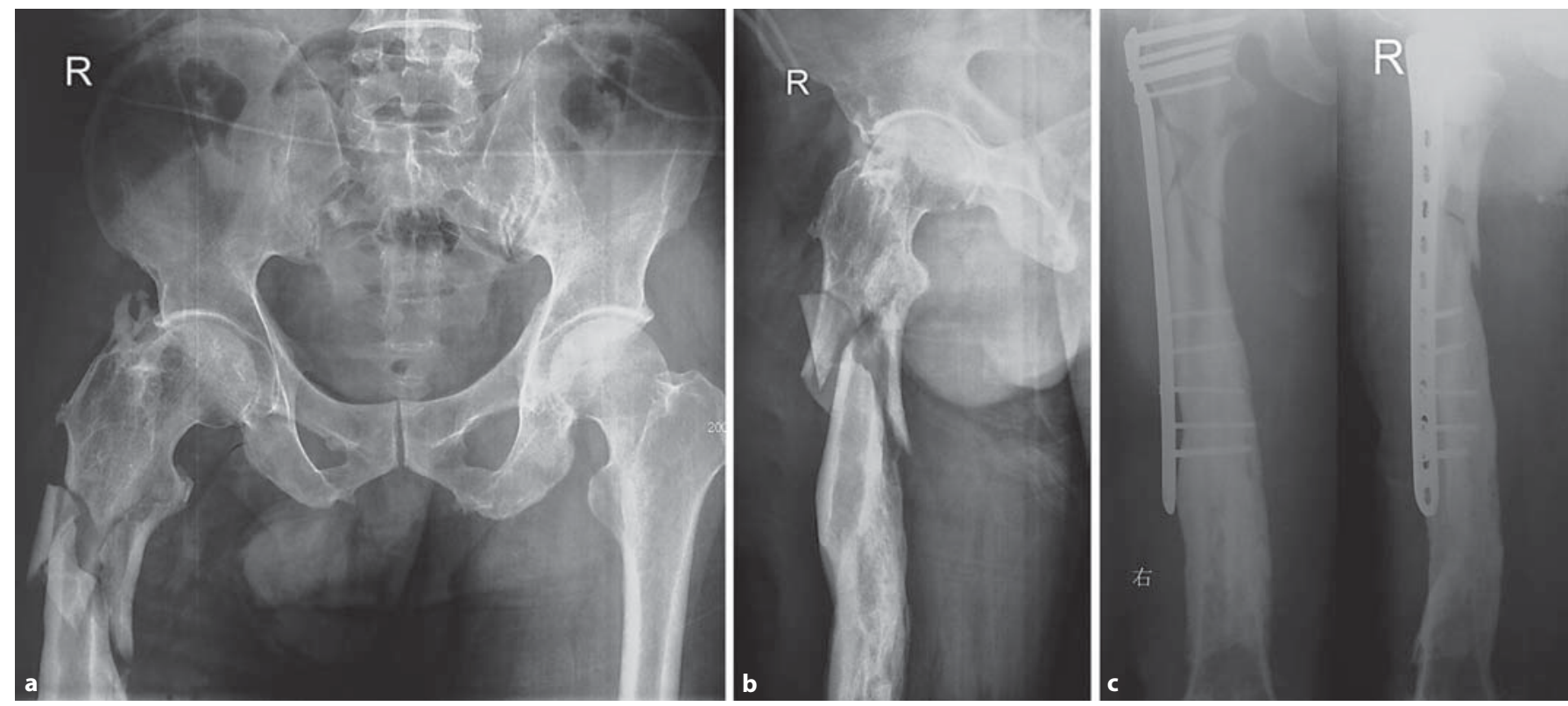

Fig. 2. a 76-year-old man with a comminuted subtrochanteric fracture and preexisting femur osteomyelitis. b Initial lateral radiograph of one representative case showing subtrochanteric fracture of the right femur. c Postoperative lateral and AP radiograph of the subtrochanteric fracture stabilized by reverse LISS plate, with an anatomic reduction and ideal implant position.
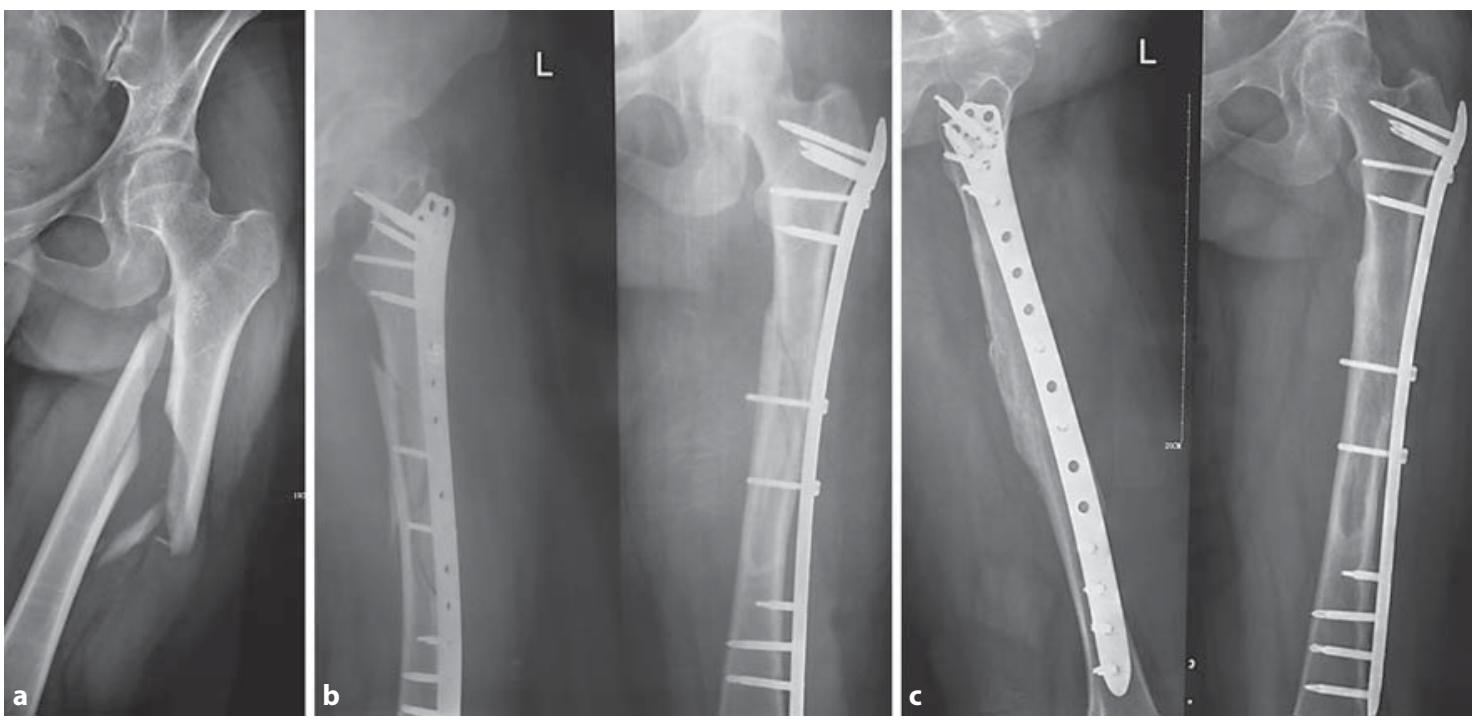

Fig. 3. a AP radiograph showing a long spiral comminuted subtrochanteric femoral fracture of the left femur in a 65-year-old woman who fell outside home. b Lateral and AP radiograph on the day following fixation. c Lateral and AP radiograph 16 weeks after fixation showing excellent union of the fracture. 
wound infection was observed. There were no collapses, cutouts, or screw penetrations, but backing out and loosening of the locking screws were observed in 2 cases. There was no reoperation. No patient had a poor functional result or failure, and all were satisfied with their postoperative functional results at the latest follow-up (fig. 2, 3).

\section{Discussion}

Fractures in the subtrochanteric region are difficult to treat because of their anatomical and biomechanical features [6]. High compressive and tensile stresses across this region are related to nonunion [7]. Since it is the transition zone between the spongious and cortical bone and its vascularization is less than in the trochanteric region, union of fractures of this site is different from the trochanteric region [8]. Furthermore, these stresses could cause internal fixations of inadequate strength to fail [9]. In the present study, we achieved a 100\% union and a low complication rate was achieved. This was possible because of the following advantages of the LISS technique. Firstly, the screws were locked with the plate to form a rigid structure, which effectively prevented screw loosening and loss of fracture reduction. This rigid structure provided a good basis, even in osteoporotic bone, for preventing the screws from sliding and pull-out. Secondly, the LISS plate could accept 4-6 screws at the proximal fragment fracture and thus provided a high pull-out resistance. Finally, fixation with a LISS plate in the lateral recumbent position decreases the tension on the iliopsoas and adductor, which facilitates spontaneous reduction of the posterior-medial fragment and reduces the stress on the proximal screws.

The LISS system was developed by the AO group for stabilization of distal femur and proximal tibial fractures according to the principles of 'minimally invasive surgery' $[10,11]$. It is an extramedullary internal fixator which combines the advantages of both interlocked intramedullary nailing techniques [12] and the early advances of the so-called biological plating technique into one system. The LISS behaves as an internal splint and is stabilized by insertion of screws which lock into the plate holes to prevent toggling of screws [13]. This is performed with the aid of an aiming device through the stab incision. The LISS plate allows percutaneous insertion and screwing via the aiming device, which is a more convenient and minimally invasive surgery. The locking screw might have the theoretical advantages of a shorter operation time, less blood loss, and fewer complications of the bony and soft tissue. The operation time was shown to affect the time to union. It might be because of the longer the operation time, the greater complexity of the fracture and the greater the severity of soft-tissue injury, both of which could delay bone healing. Furthermore, the short operating time of this method is extremely important in the elderly patient where the other diseases may take precedence and rapid fracture fixation is required.

An important consideration in this technique is that a contralateral implant was used, i.e. a left-sided implant was used for this right-sided fracture. This means that there is occasionally a misfit between the implant and the greater trochanter. When using long LISS, it is necessary to bend the distal portion to adapt the femur well. Furthermore, the position of proximal screws may lay low and horizontal. When the proximal fragment is small, it is difficult to fix the small proximal fragment. From this viewpoint, it may be better to bend the proximal portion for fixing the proximal screws toward the head obliquely.

Although all patients were treated by the same physicians in the same hospital, there were some limitations in our study. First, the sample size was relatively small and there was no control group. Therefore, significant statistical conclusions are more difficult to make. Second, some of our patients experienced discomfort and pain in the trochanteric area. Although our patients were not bothered in their daily lives by this problem, there is a need to develop a well-fitted locking plate for the proximal femur. Third, all patients appeared to have similar fracture patterns; nonetheless, there could have been differences between them, such as in the severity of osteoporosis. More biomechanical studies and prospective clinical trials are required to evaluate the validity of the locking plate for treatment of these challenging injuries.

\section{Conclusions}

This study showed that reverse LISS plating was a good technique for fixation of STF in elderly patients; no fracture collapses were observed; the surgical technique was simple and safe. Nonetheless, this procedure should not replace the use of intramedullary devices for STF under all circumstances. For situations where nailing is difficult, reverse LISS plating may be a good alternative with a high union rate and a low complication rate. 


\section{References}

1 Winquist RA, Hansen ST, Clawson DK: Closed intramedullary nailing of femoral fractures - a report of five hundred and twenty cases. J Bone Joint Surg Am 2001; 83-A:1912.

-2 Kakkar R, Kumar S, Singh A: Cephalomedullary nailing for proximal femoral fractures. Int Orthop 2005;29:21-24.

-3 Lewis JRP, Ashcroft GP: Reverse LISS plating for proximal segmental femoral fractures in the polytrauma patient: a case report. Injury 2007;38:235-239.

4 Gupta R, Mohammed AM, Alenizi EK, Ben Nekhi A: Bone mineral density in Kuwaiti patients with end-stage renal disease. Med Princ Pract 2010;20:156-158.

5 Loizou CL, McNamara I, Ahmed K, Pryor GA, Parker MJ: Classification of subtrochanteric femoral fractures. Injury 2010;41:922928
6 Fung W, Jonsson A, Buhren V, Bhandari M: Classifying intertrochanteric fractures of the proximal femur: does experience matter? Med Princ Pract 2007;16:198-202.

7 Haidukewych GJ, Berry DJ: Nonunion of fractures of the subtrochanteric region of the femur. Clin Orthop Relat Res 2004;419:185188.

8 Sims SH: Subtrochanteric femoral fractures. Orthop Clin North Am 2002;33:113-126.

9 Parker MJ, Dutta BK, Sivaji C, Pryor GA: Subtrochanteric fractures of the femur. Injury 1997;28:91-95.

10 Kregor PJ, Stannard J, Zlowodzki M, Cole PA, Alonso J: Distal femoral fracture fixation utilizing the Less Invasive Stabilization System (L.I.S.S.): the technique and early results. Injury 2001;32(suppl 3):SC32-SC47.
11 Schutz M, Muller M, Krettek C, Hontzsch D, Regazzoni P, Ganz R, Haas N: Minimally invasive fracture stabilization of distal femoral fractures with the LISS: a prospective multicenter study. Results of a clinical study with special emphasis on difficult cases. Injury 2001;32(suppl 3):SC48-SC54.

12 Pospula W, Abu Noor T: Hackethal bundle nailing with intramedullary elastic nails in the treatment of two- and three-part fractures of the proximal humerus: initial experience at Al Razi Hospital, Kuwait. Med Princ Pract 2009;18:284-288.

13 Wieser K, Babst R: Fixation failure of the LCP proximal femoral plate 4.5/5.0 in patients with missing posteromedial support in unstable per-, inter-, and subtrochanteric fractures of the proximal femur. Arch Orthop Trauma Surg 2010;130:1281-1287. 